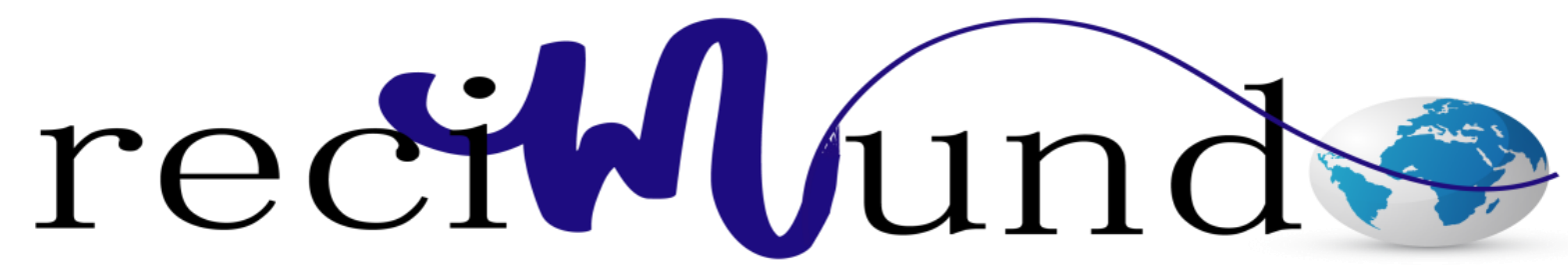

Revista Científica Mundo de la Investígación y el Conocimiento

Josselin Estefania Villamarin Barreiro a ; Andrea Desiree Escudero Requena ${ }^{\text {b; }}$

Andrea Estefania Aguirre Espinosa ${ }^{c}$; Blanca Andreina Mendoza Lino ${ }^{d}$

Insuficiencia respiratoria en pacientes con antecedentes de tabaquismo

Respiratory insufficiency in patients with a history of smoking

Revista Científica Mundo de la Investigación y el Conocimiento. Vol. 3 núm.3. Esp., noviembre, ISSN: 2588-073X, 2019, pp. 3-25

DOI: $10.26820 /$ recimundo/3.(3.Esp).noviembre.2019.3-25

URL: http://recimundo.com/index.php/es/article/view/587

Código UNESCO: 3205 Medicina Interna

Tipo de Investigación: Artículo de Revisión

(C) RECIMUNDO; Editorial Saberes del Conocimiento, 2019

Recibido: 15/09/2019

Aceptado: 23/10/2019

Publicado: 30/11/2019

Correspondencia: josselinvillamarin@gmail.com

a. Médico; Investigadora Independiente; Guayaquil, Ecuador; josselinvillamarin@gmail.com

b. Médico; Investigadora Independiente; Guayaquil, Ecuador; andreader89@ hotmail.com

c. Médico; Investigadora Independiente; Guayaquil, Ecuador; aeaguirreczs5@gmail.com

d. Médica Cirujana; Investigadora independiente; Guayaquil, Ecuador; andreinamendozalino@ hotmail.com 


\section{Prevalencia de parasitosis en habitantes de 0 a 20 años de la Parroquia El Anegado del Cantón Jipijapa}

Vol. 3, núm. 3 Esp., (2019)

Anita María Murillo Zavala; Carlos Pedro Marcillo Carvajal; Irma Gisella Parrales Pincay; Cristóbal Rolando Barcia Menéndez

\section{RESUMEN}

El tabaquismo ha sido reconocido como el factor de riesgo más determinante para enfermedad y cáncer de pulmón. Cerca del 90\% de los casos de cáncer de pulmón es causado por el hábito de fumar. El cigarrillo también es un factor causal en cáncer de nariz, boca, orofaringe, hipofaringe, laringe, esófago, estómago, páncreas, hígado, colon, cuello uterino y de leucemia mieloide. La Organización Mundial de la Salud estima que ocurren 4,9 millones de muertes anuales relacionadas con el consumo de tabaco. Mediante el proceso de una investigación documental y abarcando una metodología de revisión se logró evidenciar que el consumo de tabaco en forma frecuente y excesiva produce una serie de complicaciones a largo plazo que además resultan incurables tales como "la insuficiencia respiratoria". A nivel mundial, aproximadamente 6 millones de personas mueren al año por el consumo del tabaco, tanto por su uso directo como por humo de segunda mano. Para el 2020 se estima que esta cifra incrementará a 7.5 millones. Son ampliamente conocidas las enfermedades asociadas con esta adicción: cáncer de pulmón, 71\%; enfermedades respiratorias (incluyendo la EPOC), 42\%; y casi 10\% de enfermedades cardiovasculares, colocando al tabaquismo como el problema de salud pública más importante. La enfermedad pulmonar obstructiva crónica (EPOC) es una enfermedad pulmonar que causa episodios de dificultad para respirar, tos y producción mucosa. Estos episodios son sumamente incapacitantes; pueden durar desde unos días a varios meses y, a veces, pueden provocar la muerte. El tabaquismo activo y pasivo continúa siendo un problema grave de salud pública en el medio nacional y se asocia a mayor riesgo de desarrollar infecciones respiratorias, debido a los efectos nocivos de la nicotina y otros componentes del tabaco sobre la estructura y función del sistema respiratorio y el sistema inmune del huésped.

Palabras Claves: Insuficiencia; Respiratoria; Infección; Pulmonar; Tabaquismo. 


\title{
Prevalencia de parasitosis en habitantes de 0 a 20 años de la Parroquia El Anegado del Cantón Jipijapa
}

Vol. 3, núm. 3 Esp., (2019)

Anita María Murillo Zavala; Carlos Pedro Marcillo Carvajal; Irma Gisella Parrales Pincay;

Cristóbal Rolando Barcia Menéndez

\begin{abstract}
Shas been recognized as the most determining risk factor for lung cancer and disease. About $90 \%$ of lung cancer cases are caused by smoking. Cigarette is also a causal factor in cancer of the nose, mouth, oropharynx, hypopharynx, larynx, esophagus, stomach, pancreas, liver, colon, cervix and myeloid leukemia. The World Health Organization estimates that 4.9 million annual deaths related to tobacco use occur. Through the process of a documentary investigation and covering a review methodology, it was possible to demonstrate that the frequent and excessive consumption of tobacco produces a series of long-term complications that are also incurable such as "respiratory failure". Worldwide, approximately 6 million people die each year from tobacco use, both from direct use and secondhand smoke. By 2020 it is estimated that this figure will increase to 7.5 million. The diseases associated with this addiction are widely known: lung cancer, 71\%; respiratory diseases (including COPD), 42\%; and almost 10\% of cardiovascular diseases, placing smoking as the most important public health problem. Chronic obstructive pulmonary disease (COPD) is a lung disease that causes episodes of difficulty breathing, cough and mucous production. These episodes are extremely disabling; They can last from a few days to several months and sometimes they can cause death. Active and passive smoking continues to be a serious public health problem in the national environment and is associated with an increased risk of developing respiratory infections, due to the harmful effects of nicotine and other tobacco components on the structure and function of the respiratory system and the host's immune system
\end{abstract}

KeyWords: Insuficiencia; Respiratoria; Infección; Pulmonar; Tabaquismo. 


\section{Prevalencia de parasitosis en habitantes de 0 a 20 años de la Parroquia El Anegado del Cantón Jipijapa}

Vol. 3, núm. 3 Esp., (2019)

Anita María Murillo Zavala; Carlos Pedro Marcillo Carvajal; Irma Gisella Parrales Pincay; Cristóbal Rolando Barcia Menéndez

\section{Introducción.}

El tabaquismo ha sido reconocido como el factor de riesgo más determinante para enfermedad y cáncer de pulmón. Cerca del $90 \%$ de los casos de cáncer de pulmón es causado por el hábito de fumar. El cigarrillo también es un factor causal en cáncer de nariz, boca, orofaringe, hipofaringe, laringe, esófago, estómago, páncreas, hígado, colon, cuello uterino y de leucemia mieloide. El tabaco también es una de las causas más importantes de infarto agudo de miocardio (IAM), especialmente en hombres. Según un estudio, fumar incrementa 3 veces el riesgo de presentar IAM; intervalo de confianza del 95\%, riesgo que aumenta en proporción al número de cigarrillos fumados. Otra de las afecciones de importancia en salud pública, es la enfermedad pulmonar obstructiva crónica (EPOC), la cual aporta gran carga de morbilidad y mortalidad, sin mencionar los altos costos para el sistema de salud y las repercusiones en la calidad de vida de los pacientes. El principal factor de riesgo para la aparición de EPOC es el tabaquismo, el 80\% de los pacientes con esta enfermedad tiene antecedente de consumo de cigarrillos. (Martinez \& Diaz, 2013)

El tabaquismo es el principal factor de riesgo de muerte prematura prevenible en el mundo. Según la obra de Saldias, Mendez, Ramirez, y Diaz (2007) mensionan que la Organización Mundial de la Salud estima que ocurren 4,9 millones de muertes anuales relacionadas con el consumo de tabaco. El consumo activo y pasivo de tabaco tiene efectos deletéreos sobre múltiples órganos, especialmente el sistema respiratorio. El cáncer bronquial y la enfermedad pulmonar obstructiva crónica son las principales enfermedades respiratorias 


\section{Prevalencia de parasitosis en habitantes de 0 a 20 años de la Parroquia El}

Anegado del Cantón Jipijapa

Vol. 3, núm. 3 Esp., (2019)

Anita María Murillo Zavala; Carlos Pedro Marcillo Carvajal; Irma Gisella Parrales Pincay;

Cristóbal Rolando Barcia Menéndez

asociadas al tabaquismo. En las últimas décadas, se ha reconocido la importancia del tabaquismo pasivo en la salud del niño.

Estos mismos expresan que la exposición prolongada a humo de tabaco también afecta la incidencia, gravedad y evolución de múltiples otras afecciones respiratorias, como resfrío común, influenza, neumonía, tuberculosis, neumotórax, hemorragia pulmonar y algunas enfermedades intersticiales. Los mecanismos patogénicos por los cuales el consumo de tabaco aumenta la incidencia y gravedad de algunas enfermedades respiratorias no han sido completamente esclarecidos. El tabaquismo activo y la exposición a humo de tabaco (fumador pasivo) aumentan significativamente el riesgo de infecciones respiratorias, lo cual no es ampliamente reconocido por el equipo médico. En este artículo revisaremos los trastornos fisiológicos y morfológicos ocasionados por el consumo de tabaco sobre el sistema respiratorio y el sistema inmune, que podrían explicar el aumento del riesgo de padecer infecciones respiratorias en la etapa infantil y adulta.

Así mismo se entiende que el consumo de tabaco en forma frecuente y excesiva produce una serie de complicaciones a largo plazo que además resultan incurables. Destacando también el hecho de que aparte de las complicaciones respiratorias ya mencionadas se producen otros problemas en otros sistemas del cuerpo.

\section{Materiales y Métodos.}

Las herramientas y materiales se conciben para el desarrollo de este trabajo investigativo son los siguientes: computadores personales con conexión a internet y un cúmulo de contenidos 


\section{Prevalencia de parasitosis en habitantes de 0 a 20 años de la Parroquia El Anegado del Cantón Jipijapa}

Vol. 3, núm. 3 Esp., (2019)

Anita María Murillo Zavala; Carlos Pedro Marcillo Carvajal; Irma Gisella Parrales Pincay; Cristóbal Rolando Barcia Menéndez

científico académicos diversos, con el fin de aportar información de calidad y actualizada respecto a la Insuficiencia respiratoria en pacientes con antecedentes de tabaquismo. Se escoge un diseño documental, de tipo no experimental, de un nivel descriptivo.

Esta investigación se enfoca en la búsqueda y revisión sistemática de literatura científicoacadémica seleccionada, que por una parte, está disponible determinadas bases de datos, entre las que figuran: MedlinePlus, PubMed, Biblioteca Virtual de la Salud (BVS), SciELO, Dialnet y ELSEVIER, Cochrane, entre otras; y por la otra, mediante el uso del material físicamente disponible, representando todo esto parte esencial del proceso investigativo y de comprensión que facilita la síntesis de la mejor evidencia disponible, y a su vez representando todo ello uno de los resultados esperados.

Principalmente se realiza una búsqueda aleatoria y consecutiva en las mencionadas bases de datos, usando las expresiones “insuficiencia respiratoria por tabaquismo", “infección respiratoria por tabaquismo", "enfermedades respiratorias por tabaquismo" y "EPOC", lo que aproximadamente resultó en miles de registros bibliográficos. Luego éstos se filtran en base a criterios de: idioma español, relevancia, correlación temática y fecha de publicación en los últimos 10 años, salvo alguna excepciones, sin descartar por tipo de material bibliográfico; es decir, se escogen títulos de artículos científicos, ensayos, revisiones sistemáticas, libros, boletines, folletos, tesis de grado, posgrado y doctorado, noticias científicas, entre otros documentos e información de interés científico y académico, descartando así, editoriales, cartas al editor, estudios de corte entre otros. 


\section{Prevalencia de parasitosis en habitantes de 0 a 20 años de la Parroquia El}

Anegado del Cantón Jipijapa

Vol. 3, núm. 3 Esp., (2019)

Anita María Murillo Zavala; Carlos Pedro Marcillo Carvajal; Irma Gisella Parrales Pincay;

Cristóbal Rolando Barcia Menéndez

Paralelamente, se adelantó una búsqueda sin limitación cronológica ni de nivel de evidencia para identificar información igualmente relevante y necesaria para el desarrollo de la presente temática y revisión, siendo a partir de allí que el equipo investigador le da la correspondiente lectura crítica y análisis interpretativo a toda la evidencia en definitiva seleccionada, resultando dicho proceso en el fundamento de las ideas aquí expresadas de manera consensuada.

La inclusión o exclusión de cada una de las referencias en la presente revisión se definió igualmente por el consenso todos los participantes en este proceso investigativo, y de la misma manera fueron resueltas las decisiones desiguales.

\section{Resultados.}

A nivel mundial, aproximadamente 6 millones de personas mueren al año por el consumo del tabaco, tanto por su uso directo como por humo de segunda mano. Para el 2020 se estima que esta cifra incrementará a 7.5 millones. Son ampliamente conocidas las enfermedades asociadas con esta adicción: cáncer de pulmón, 71\%; enfermedades respiratorias, 42\%; y casi $10 \%$ de enfermedades cardiovasculares, colocando al tabaquismo como el problema de salud pública más importante.

En América Latina mueren más de 400 personas cada día como consecuencia del tabaquismo. A la fecha, se estima que hay 150 mil muertes anuales en América Latina y el Caribe atribuidas al uso de este producto. Tan solo en México casi 68 de cada 100 personas de 18 a 29 años de edad consumió tabaco alguna vez en su vida, provocando problemas no sólo 


\section{Prevalencia de parasitosis en habitantes de 0 a 20 años de la Parroquia El Anegado del Cantón Jipijapa}

Vol. 3, núm. 3 Esp., (2019)

Anita María Murillo Zavala; Carlos Pedro Marcillo Carvajal; Irma Gisella Parrales Pincay; Cristóbal Rolando Barcia Menéndez

relacionados a la salud, sino también sociales debido a los costos del consumo, el medio ambiente y la calidad de vida del individuo fumador y quien vive con él. En México, para el año 2020 el tabaco estará ocasionando el fallecimiento de 400 mil personas cada año. Datos de la Organización Panamericana de la Salud (OPS) de 2005 indican que la proporción de consumo de tabaco en adultos en el país es del 8.3\%. Este porcentaje coloca a México por encima de países como Ecuador, Guatemala y Costa Rica con $4.8 \%, 4.1 \%$ y $3.0 \%$, respectivamente.

La Encuesta Nacional de Salud y Nutrición 2006 señala que casi 47 de cada 100 varones de 20 años a nivel nacional, han fumado al menos 100 cigarrillos durante su vida, mientras que poco más de 15 de cada 100 mujeres comparte esa característica. Asimismo, el número de personas de 18 a 65 años que fuma diariamente representa $54.1 \%$ del total de fumadores; más de medio millón consume 20 y más cigarrillos al día. Para ambos sexos, más de $60 \%$ fuma entre uno y cinco cigarrillos diarios.

Estos datos reflejan que el consumo del tabaco y la exposición a su humo se mantienen como la primera causa de muerte prevenible en el mundo, tanto de fumadores activos, como a causa del humo pasivo. El problema del consumo de tabaco no sólo reside en las muertes a las que se asocia, sino también a la disminuida calidad de vida que se observa en los fumadores debido al deterioro y las complicaciones que ocasionan las comorbilidades como: cáncer de pulmón, laringe, riñón, vejiga, estómago, colon, cavidad oral, esófago, enfermedad pulmonar obstructiva crónica (EPOC), asma, diabetes, cardiopatía isquémica, aborto, parto prematuro, problemas del estado de ánimo como depresión mayor y ansiedad, problemas cognitivos, etc. Una de las graves consecuencias del tabaquismo son las enfermedades respiratorias, entre ellas la 


\section{Prevalencia de parasitosis en habitantes de 0 a 20 años de la Parroquia El}

Anegado del Cantón Jipijapa

Vol. 3, núm. 3 Esp., (2019)

Anita María Murillo Zavala; Carlos Pedro Marcillo Carvajal; Irma Gisella Parrales Pincay;

Cristóbal Rolando Barcia Menéndez

EPOC que representa una fuerte disminución en la calidad de vida del paciente por la cronicidad de los síntomas y la necesidad de hospitalizaciones en caso de exacerbación. En México, aproximadamente el $70 \%$ de los casos de EPOC se asocian al tabaquismo y el restante a la exposición crónica al humo de biomasa. Esto no quiere decir que todos los fumadores desarrollan EPOC (alrededor del 15 y $20 \%$ de ellos), sino que la principal causa de la enfermedad es el tabaquismo. La prevalencia mundial de la EPOC en sujetos mayores de 45 años se estima que puede ser de 3 a 10\%, lo cual la coloca en el cuarto lugar de causa de muertes a nivel global, junto con el sida. En el país tiene una prevalencia alrededor del 7.8\%9 y ocupa el cuarto lugar en mujeres y el quinto en hombres dentro de las 10 primeras causas de mortalidad general. (Diaz, Garcia, Sansores, \& Ramirez, 2014)

¿Qué es la insuficiencia respiratoria?

La insuficiencia respiratoria es una afección en la cual su sangre no tiene suficiente oxígeno o tiene demasiado dióxido de carbono. A veces puede tener ambos problemas. Cuando respira, sus pulmones se llenan de oxígeno. El oxígeno pasa a su sangre, que lo lleva a sus órganos, como el corazón y el cerebro, que necesitan sangre rica en oxígeno para funcionar bien. Otra función de la respiración es eliminar el dióxido de carbono de la sangre al botar el aire. Tener demasiado dióxido de carbono en la sangre puede dañar sus órganos. (MedlinePlus, 2019)

La insuficiencia respiratoria puede clasificarse de diversas formas: según la rapidez de instauración, los gases sanguíneos, las características anatomo-funcionales del aparato respiratorio y el mecanismo fisiopatológico. La insuficiencia respiratoria aguda (IRA) se instaura en pocas horas y se produce sobre un pulmón sano. La insuficiencia respiratoria crónica (IRC) se 


\section{Prevalencia de parasitosis en habitantes de 0 a 20 años de la Parroquia El Anegado del Cantón Jipijapa}

Vol. 3, núm. 3 Esp., (2019)

Anita María Murillo Zavala; Carlos Pedro Marcillo Carvajal; Irma Gisella Parrales Pincay; Cristóbal Rolando Barcia Menéndez

instaura en meses o años y se produce en un pulmón patológico. También puede producirse agudización de una IRC. En el pulmón sano, la insuficiencia respiratoria hipercápnica se produce por hipoventilación. En el pulmón patológico la insuficiencia respiratoria, tanto hipoxémica como hipercánica, responde a diversos mecanismos fisiopatológicos. (Fundacion para la formacion e investigacion sanitarias de la region de Murcias)

Clasificación de la IRA

$>$ Tipo I o hipoxémico: caracterizado por la existencia de hipoxemia asociada a cifras de $\mathrm{PaCO} 2$ normales o disminuidas. Se produce como consecuencia de la disminución de la relación V/Q o la presencia de shunt, y en aquellas enfermedades que alteran la difusión. El desarrollo de acidosis (metabólica) implica disfunción de otrosórganos por hipoperfusión.

$>$ Tipo II o hipercápnico: caracterizado por el aumento arterial del CO2. Es menos frecuente en niños y pese a que puede tratarse de una situación crónica, también puede seguir a una IRA tipo I cuando los músculos respiratorios se fatigan y son incapaces de mantener el VM. Se debe a la disfunción de las estructuras encargadas de la ventilación: las vías aéreas, los bronquios, la caja torácica, los músculos respiratorios o el centro respiratorio, a pesar de lo cual el pulmón puede 


\section{Prevalencia de parasitosis en habitantes de 0 a 20 años de la Parroquia El}

Anegado del Cantón Jipijapa

Vol. 3, núm. 3 Esp., (2019)

Anita María Murillo Zavala; Carlos Pedro Marcillo Carvajal; Irma Gisella Parrales Pincay;

Cristóbal Rolando Barcia Menéndez

estar completamente sano o no. No mejoran con oxigenoterapia y precisan ventilación mecánica. (Pastor, Perez, \& Rodriguez, 2017)

$I R C$

Existen múltiples causas de la insuficiencia respiratoria crónica que se originan en los diferentes elementos que forman parte del aparato respiratorio. Los síntomas de la insuficiencia respiratoria crónica derivan de la hipoxemia y la hipercapnia como: disnea, hipertensión arterial secundaria a la vasoconstricción sistémica, hipertensión arterial pulmonar, agitación y cefalea; en fases avanzadas aparece coma hipercápnico, poliglobulia, cor pulmonale y fenómenos tromboembólicos. (Caceres, Vasquez, Macos, \& Gomez, 2014)

Formas en que el tabaco pone en peligro la salud pulmonar de las personas de todo el mundo:

Cáncer de pulmón. Fumar tabaco es la principal causa del cáncer de pulmón, responsable de más de dos tercios de las muertes por cáncer de pulmón en todo el mundo. La exposición al humo de tabaco ajeno en el hogar o en el lugar de trabajo también aumenta el riesgo de cáncer de pulmón. Dejar de fumar puede reducir el riesgo de cáncer de pulmón: después de 10 años de dejar de fumar, el riesgo de cáncer de pulmón se reduce a aproximadamente la mitad del de un fumador.

Enfermedades respiratorias crónicas. Fumar tabaco es la causa principal de la EPOC, una afección en que la acumulación de mucosidad con pus en los pulmones provoca una tos dolorosa y una terrible insuficiencia respiratoria. El 


\section{Prevalencia de parasitosis en habitantes de 0 a 20 años de la Parroquia El}

Anegado del Cantón Jipijapa

Vol. 3, núm. 3 Esp., (2019)

Anita María Murillo Zavala; Carlos Pedro Marcillo Carvajal; Irma Gisella Parrales Pincay; Cristóbal Rolando Barcia Menéndez

riesgo de desarrollar EPOC es particularmente alto entre las personas que comienzan a fumar a una edad temprana, ya que el humo del tabaco retrasa significativamente el desarrollo pulmonar. El tabaco también exacerba el asma, que restringe la actividad y contribuye a la discapacidad. El abandono temprano del hábito de fumar es el tratamiento más eficaz para retrasar la progresión de la EPOC y mejorar los síntomas del asma.

A lo largo de la vida. Los bebés expuestos en el útero a las toxinas del humo de tabaco, a través del tabaquismo materno o de la exposición materna al humo ajeno, experimentan con frecuencia una disminución del crecimiento de los pulmones y de la función pulmonar. Los niños pequeños expuestos al humo de tabaco ajeno corren el riesgo de agravamiento del asma, la neumonía y la bronquitis, así como infecciones frecuentes de las vías respiratorias inferiores.

A nivel mundial, se calcula que 165000 niños mueren antes de cumplir 5 años por infecciones de las vías respiratorias inferiores causadas por el humo de tabaco ajeno. Los que viven hasta la edad adulta siguen sufriendo las consecuencias para la salud de la exposición al humo de tabaco ajeno, ya que las infecciones frecuentes de las vías respiratorias inferiores en la primera infancia aumentan significativamente el riesgo de desarrollar EPOC en la edad adulta.

Tuberculosis. La tuberculosis (TB) daña los pulmones y reduce la función pulmonar, lo que se ve agravado por el tabaquismo. Los componentes químicos del humo del tabaco pueden desencadenar infecciones latentes de TB, con las que 


\section{Prevalencia de parasitosis en habitantes de 0 a 20 años de la Parroquia El}

Anegado del Cantón Jipijapa

Vol. 3, núm. 3 Esp., (2019)

Anita María Murillo Zavala; Carlos Pedro Marcillo Carvajal; Irma Gisella Parrales Pincay;

Cristóbal Rolando Barcia Menéndez

está infectada alrededor de una cuarta parte de la población. La TB activa, agravada por los efectos nocivos del tabaquismo en la salud pulmonar, aumenta sustancialmente el riesgo de discapacidad y muerte por insuficiencia respiratoria.

$>$ Contaminación del aire. El humo del tabaco es una forma muy peligrosa de contaminación del aire de interiores: contiene más de 7000 sustancias químicas, 69 de las cuales se sabe que causan cáncer. Aunque el humo puede ser invisible e inodoro, puede permanecer en el aire hasta cinco horas, poniendo a las personas expuestas en riesgo de desarrollar cáncer de pulmón, enfermedades respiratorias crónicas y reducción de la función pulmonar. (Organizacion Mundial de la Salud, 2019)

Riesgos para la salud debido al tabaquismo

En los Estados Unidos, aproximadamente la mitad de todas las personas que continúan fumando morirán debido al hábito. Más de 480,000 personas mueren cada año en este país a causa de enfermedades relacionadas con el consumo de tabaco. Esto significa que cada año el fumar causa aproximadamente 1 de cada 5 muertes en los Estados Unidos.

$>$ El hábito de fumar cigarrillos mata a más estadounidenses que el alcohol, los accidentes automovilísticos, el SIDA, las armas de fuego, y las drogas ilegales en conjunto.

Los fumadores de cigarrillos mueren más jóvenes que los no fumadores. 


\section{Prevalencia de parasitosis en habitantes de 0 a 20 años de la Parroquia El Anegado del Cantón Jipijapa}

Vol. 3, núm. 3 Esp., (2019)

Anita María Murillo Zavala; Carlos Pedro Marcillo Carvajal; Irma Gisella Parrales Pincay; Cristóbal Rolando Barcia Menéndez

$>$ Fumar acorta la vida de fumadores masculinos por unos 12 años y la vida de las fumadoras por aproximadamente 11 años.

Fumar no sólo causa cáncer, sino que puede ocasionar daño a casi todos los órganos en el cuerpo, incluyendo los pulmones, el corazón, los vasos sanguíneos, los órganos genitales, la boca, la piel, los ojos y los huesos.

Fumar daña las vías respiratorias y los pequeños sacos de aire en sus pulmones. Este daño comienza temprano en los fumadores, y la función pulmonar continúa empeorando mientras la persona fume. Sin embargo, puede que tome años antes de que se pueda notar el problema lo suficientemente como para diagnosticar la enfermedad pulmonar. Asimismo, fumar puede ocasionar que la pulmonía y el asma empeoren. Además, causa muchas otras enfermedades pulmonares que pueden ser casi tan graves como el cáncer de pulmón. (Sociedad America del Cancer, 2018)

\section{Infecciones respiratorias en fumadores}

El humo del tabaco, a través de sus partículas tóxicas, incide en el aparato respiratorio de los fumadores activos y pasivos, produciendo alteración tanto en la estructura como en la función de las células, así como en la inmunidad, originando enfermedades e infecciones respiratorias. El efecto adverso del humo del tabaco sobre el aparato respiratorio sería el siguiente:

1. Vías aéreas centrales:

$>$ Pérdida de epitelio ciliar y disminución del aclaramiento mucociliar. 


\section{Prevalencia de parasitosis en habitantes de 0 a 20 años de la Parroquia El}

Anegado del Cantón Jipijapa

Vol. 3, núm. 3 Esp., (2019)

Anita María Murillo Zavala; Carlos Pedro Marcillo Carvajal; Irma Gisella Parrales Pincay;

Cristóbal Rolando Barcia Menéndez

Hiperplasia de glándulas mucosas y aumento del número de células caliciformes.

Cambio del epitelio ciliado pseudoestratificado a epitelio no ciliado.

2. Vías aéreas periféricas:

Edema epitelial, metaplasia de células caliciformes, impactación mucosa, fibrosis peribronquial, hipertrofia de la capa muscular.

3. Alveolos y capilares:

$>$ Engrosamiento y esclerosis de las células alveolares, destrucción de los alveolos peribronquiolares, engrosamiento de los capilares pulmonares, pérdida del lecho vascular periférico. Activación de neutrófilos y de macrófagos.

4. Defensa celular pulmonar:

Descenso de la capacidad de activación de los linfocitos bronquiales.

Disminución de la movilidad de los neutrófilos y de los macrófagos, y éstos con disminución de la adherencia y capacidad fagocítica.

Aumento de la liberación de sustancias lesivas (elastasa).

5. Respuesta inmunitaria alterada:

Aumento del número de eosinófilos en sangre periférica.

Disminución de la respuesta inmune a los antígenos inhalados. 


\section{Prevalencia de parasitosis en habitantes de 0 a 20 años de la Parroquia El Anegado del Cantón Jipijapa}

Vol. 3, núm. 3 Esp., (2019)

Anita María Murillo Zavala; Carlos Pedro Marcillo Carvajal; Irma Gisella Parrales Pincay; Cristóbal Rolando Barcia Menéndez

La exposición a humo de tabaco constituye un importante factor de riesgo para adquirir infecciones respiratorias bacterianas y virales.

En adultos sin enfermedad pulmonar obstructiva crónica, el tabaquismo está asociado a un aumento significativo del riesgo de neumonía, enfermedad neumocócica invasiva e infección por Legionella spp. Además, el tabaquismo está asociado a mayor riesgo de influenza, tuberculosis y neumonía por virus varicela. (Diaz., 2017)

\section{Función respiratoria}

Tanto en hombres como en mujeres los indicadores espirométricos fueron significativamente más bajos en las personas que fuman en comparación con quienes no fuman, las diferencias varían entre $2 \%$ y $15 \%$.

El flujo espiratorio al $25 \%$ de la capacidad vital presentó diferencias cercanas al $12 \%$ a favor de las personas que no fuman. Los flujos espiratorios al 50\% y $75 \%$ de la capacidad vital, revelaron diferencias de entre el $8 \%$ y $10 \%$, con valores funcionales siempre más bajos en los fumadores. La prueba más tradicional de alteraciones funcionales, es decir, la prueba de Tiffeneau mostró una diferencia desfavorable para quienes respiran aire contaminado con humo de cigarrillo, cuyo promedio es 81.4 en los hombres y 82.5 en las mujeres, en comparación con las personas que no fuman, cuyos promedios son 83.4 y 84.8 , respectivamente.

Las diferencias entre personas expuestas a la inhalación de aire contaminado por humo de cigarrillo se reflejaron en todos los indicadores de función pulmonar. Estas diferencias 


\section{Prevalencia de parasitosis en habitantes de 0 a 20 años de la Parroquia El}

Anegado del Cantón Jipijapa

Vol. 3, núm. 3 Esp., (2019)

Anita María Murillo Zavala; Carlos Pedro Marcillo Carvajal; Irma Gisella Parrales Pincay;

Cristóbal Rolando Barcia Menéndez

registradas para la muestra global fueron, en magnitud y en sentido, bastante similares para los hombres y las mujeres. (Martinez \& Diaz, 2013)

EPOC

La EPOC es una enfermedad pulmonar que causa episodios de dificultad para respirar, tos y producción mucosa. Estos episodios son sumamente incapacitantes; pueden durar desde unos días a varios meses y, a veces, pueden provocar la muerte. En el 2016, se estimó que más de 251 millones de personas sufrían de EPOC.

El consumo de tabaco es el factor de riesgo más importante de EPOC, puesto que causa inflamación y ruptura de los alvéolos pulmonares, lo que reduce la capacidad de los pulmones de absorber oxígeno y expulsar dióxido de carbono. También causa la acumulación de moco purulento en los pulmones, dando lugar a tos y dificultades respiratorias agudas.

Las personas adultas que estuvieron expuestas al humo de tabaco durante su niñez y que, como consecuencia, tuvieron infecciones frecuentes de las vías respiratorias inferiores corren el riesgo de desarrollar EPOC. Las personas que comenzaron a fumar en su adolescencia o juventud son especialmente susceptibles a desarrollar EPOC como resultado de la disminución del crecimiento de los pulmones y de la función pulmonar. La mayoría de los casos de EPOC pueden prevenirse evitando el consumo de tabaco o dejando de fumar tempranamente. Los pacientes con EPOC que dejan de fumar mejoran la función pulmonar y sufren menos efectos a largo plazo. (OMS, 2019) 


\section{Prevalencia de parasitosis en habitantes de 0 a 20 años de la Parroquia El Anegado del Cantón Jipijapa}

Vol. 3, núm. 3 Esp., (2019)

Anita María Murillo Zavala; Carlos Pedro Marcillo Carvajal; Irma Gisella Parrales Pincay; Cristóbal Rolando Barcia Menéndez

La enfermedad pulmonar obstructiva crónica o EPOC, hace referencia a un grupo de afecciones que obstruyen la circulación del aire y causan problemas relacionados con la respiración. Entre estas enfermedades se encuentran el enfisema, la bronquitis crónica y, en algunos casos, el asma. (Deartamento de salud y servicios humanos, 2019)

Es causada por la exposición a largo plazo a gases o partículas irritantes, en la mayoría de los casos del humo de cigarrillo. Las personas con EPOC tienen mayor riesgo de padecer enfermedades cardíacas, cáncer de pulmón y una variedad de otras afecciones.

El enfisema y la bronquitis crónica son las dos afecciones más frecuentes que contribuyen a desarrollar la EPOC. La bronquitis crónica es la inflamación del revestimiento de los bronquios, que llevan aire hacia y desde los sacos de aire (alvéolos) del pulmón. Se caracteriza por la tos diaria y la producción de mucosidad (esputo).

El enfisema es una afección en la cual los alvéolos que están en los extremos de las vías aéreas más pequeñas (bronquiolos) de los pulmones se destruyen como resultado de la exposición dañina al humo de cigarrillo y a otros gases y partículas irritantes.

La EPOC se puede tratar. Con el tratamiento adecuado, la mayoría de las personas con EPOC puede lograr un buen control de los síntomas y la calidad de vida, además de reducir el riesgo de otras afecciones relacionadas. (MayoClinic, 2017)

\section{Síntomas}

La EPOC puede causar una variedad de síntomas, que incluyen: 


\section{Prevalencia de parasitosis en habitantes de 0 a 20 años de la Parroquia El}

Anegado del Cantón Jipijapa

Vol. 3, núm. 3 Esp., (2019)

Anita María Murillo Zavala; Carlos Pedro Marcillo Carvajal; Irma Gisella Parrales Pincay;

Cristóbal Rolando Barcia Menéndez

$>$ Una tos permanente.

Una tos que produce mucosidad.

Falta de aliento, especialmente durante la actividad física.

$>$ Una sensación apretada en el pecho.

No poder respirar profundamente.

Los síntomas de la EPOC comienzan lentamente. Empeoran durante un período de años si no se diagnostican y tratan temprano. Si se retrasa el diagnóstico y el tratamiento pueden provocarse complicaciones. Estas podrían incluir problemas cardíacos (latidos cardíacos irregulares e insuficiencia cardíaca), presión arterial alta e infecciones respiratorias. Las infecciones pueden dañar aún más sus pulmones. (¿Qué es la enfermedad pulmonar obstructiva crónica (EPOC)?, 2019)

\section{Diagnostico}

Para diagnosticar la EPOC el neumólogo, aparte del examen físico y la descripción de síntomas, encargará una analítica de sangre de bioquímica completa y hemograma para recuento de leucocitos y una espirometría. Esta es la prueba de función pulmonar más habitual, en la que se mide la cantidad de aire que pueden retener los pulmones y a qué velocidad sale de ellos. También se solicitará una radiografía torácica y una tomografía computarizada. 


\section{Prevalencia de parasitosis en habitantes de 0 a 20 años de la Parroquia El Anegado del Cantón Jipijapa}

Vol. 3, núm. 3 Esp., (2019)

Anita María Murillo Zavala; Carlos Pedro Marcillo Carvajal; Irma Gisella Parrales Pincay; Cristóbal Rolando Barcia Menéndez

Aparte, se hace una gasometría arterial para medir la presión de oxígeno y dióxido de carbono en una muestra de sangre. Esta muestra se obtiene de una arteria, normalmente, de la arteria radial situada en la muñeca. Esta prueba requiere anestesia local.

Otra prueba es el test de marcha de 6 minutos que mide la distancia que puede recorrer una persona en ese tiempo. También se estudia la función cardíaca para lo que se suele hacer un ecocardiograma. (La Vanguardia, 2019)

\section{Conclusiones.}

La EPOC y el tabaquismo tienen un impacto importante sobre el funcionamiento cognitivo, particularmente por la vulnerabilidad de algunas estructuras cerebrales a la hipoxemia, lo que desencadena una serie de eventos fisiológicos que tienen como resultado la afectación del funcionamiento cognitivo de las personas que fuman y/o con EPOC principalmente en memoria

(a corto plazo), atención, habilidades visomotrices y funciones ejecutivas, particularmente en flexibilidad cognitiva. (Diaz, Garcia, Sansores, \& Ramirez, 2014)

El tabaquismo activo y pasivo continúa siendo un problema grave de salud pública en el medio nacional y se asocia a mayor riesgo de desarrollar infecciones respiratorias, debido a los efectos nocivos de la nicotina y otros componentes del tabaco sobre la estructura y función del sistema respiratorio y el sistema inmune del huésped. Los hallazgos de esta revisión nos permiten destacar la importancia que tiene promover el cese del hábito tabáquico en la población, con el propósito de reducir el riesgo de adquirir infecciones bacterianas y virales en los individuos expuestos al humo de tabaco. Como medidas preventivas adicionales, se debería 


\section{Prevalencia de parasitosis en habitantes de 0 a 20 años de la Parroquia El Anegado del Cantón Jipijapa}

Vol. 3, núm. 3 Esp., (2019)

Anita María Murillo Zavala; Carlos Pedro Marcillo Carvajal; Irma Gisella Parrales Pincay;

Cristóbal Rolando Barcia Menéndez

recomendar la vacunación anti influenza y antineumocócica en todos los fumadores activos que no logran abandonar el hábito. (Saldias, Mendez, Ramirez, \& Diaz, 2007)

\section{Bibliografía.}

¿Qué es la enfermedad pulmonar obstructiva crónica (EPOC)? (09 de Octubre de 2019). Recuperado de American Academy of Family Physicians: https://es.familydoctor.org/condicion/enfermedad-pulmonar-obstructiva-cronica/

Caceres, L., Vasquez, E., Macos, C., \& Gomez, R. (15 de Octubre de 2014). Insuficiencia respiratoria cronica. Etiopatogenia. Clinica. Diagnostico. Medicine - Programa de Formación Médica , 11(63), 3735-3741.

Deartamento de salud y servicios humanos. (01 de Abril de 2019). El tabaquismo y la enfermedad pulmonar obstructiva crónica (EPOC). Recuperado de: https://www.cdc.gov/tobacco/campaign/tips/spanish/enfermedades/tabaquismoenfermedad-pulmonar-obstructiva-cronica.html

Diaz, N., Garcia, L., Sansores, R., \& Ramirez, A. (Julio-Septiembre de 2014). Impacto del tabaquismo y la EPOC sobre el funcionamiento cerebral. Neumología y cirugía de tórax, 73(3), 196-204.

Fundacion para la formacion e investigacion sanitarias de la region de Murcias. (s.f.). $\begin{array}{lll}\text { Insuficiencia } & \text { respiratoria. }\end{array}$ http://www.ffis.es/volviendoalobasico/21insuficiencia_respiratoria.html

La Vanguardia. (31 de Mayo de 2019). ¿Qué es la EPOC? | Síntomas, causas y cómo tratar la enfermedad. Recuperado de: https://www.lavanguardia.com/vida/salud/enfermedades- 


\section{Prevalencia de parasitosis en habitantes de 0 a 20 años de la Parroquia El Anegado del Cantón Jipijapa}

Vol. 3, núm. 3 Esp., (2019)

Anita María Murillo Zavala; Carlos Pedro Marcillo Carvajal; Irma Gisella Parrales Pincay; Cristóbal Rolando Barcia Menéndez

respiratorias/20190531/462576732811/epoc-emfermedad-pulmonar-obstructiva-cronica-

insuficiencia-respiracion-tabaquismo-fumar.html

Martinez, E., \& Diaz, P. (2013). Tabaquismo y disminución de la función pulmonar en hombres y mujeres adultos . Sociedad iberoamericana de informacion cientifica, 20(1), 246-250.

MayoClinic. (11 de Agosto de 2017). Enfermedad pulmonar obstructiva crónica (EPOC). Recuperado de: https://www.mayoclinic.org/es-es/diseases-conditions/copd/symptomscauses/syc-20353679

MedlinePlus. (25 de Julio de 2019). Insuficiencia respiratoria. Recuperado el 15 de 10 de 2019, de MedlinePlus: https://medlineplus.gov/spanish/respiratoryfailure.html

OMS. (31 de Mayo de 2019). Elige salud. Recuperado de: https://www.who.int/docs/defaultsource/world-no-tobacco-day/6188-whontd19-brochure-es-meo20190522.pdf

Organizacion Mundial de la Salud. (31 de Mayo de 2019). Día Mundial sin Tabaco 2019. Recuperado de: https://www.who.int/es/news-room/events/detail/2019/05/31/defaultcalendar/world-no-tobacco-day

Pastor, D., Perez, S., \& Rodriguez, J. (2017). Fracaso respiratorio agudo y crónico. Oxigenoterapia. Protocolo, diagnostico,terapia pediatrica, 1, 369-399.

Saldias, F., Mendez, I., Ramirez, D., \& Diaz, O. (Septiembre de 2007). Riesgo de enfermedades respiratorias en el fumador activo y pasivo. Revista chilena de enfermedades respiratorias, 23(3), 179-187.

Sociedad America del Cancer. (15 de Noviembre de 2018). Riesgos para la salud debido al tabaquismo. Recuperado de: https:/www.cancer.org/es/cancer/causas-del-cancer/tabacoy-cancer/riesgos-para-la-salud-debido-al-tabaquismo.html 
Prevalencia de parasitosis en habitantes de 0 a 20 años de la Parroquia El Anegado del Cantón Jipijapa Vol. 3, núm. 3 Esp., (2019) Anita María Murillo Zavala; Carlos Pedro Marcillo Carvajal; Irma Gisella Parrales Pincay; Cristóbal Rolando Barcia Menéndez

\section{(c) $(9)$ \\ RECONOCIMIENTO-NOCOMERCIAL-COMPARTIRIGUAL \\ CC BY-NC-SA \\ ESTA LICENCIA PERMITE A OTROS ENTREMEZCLAR, AJUSTAR Y CONSTRUIR A PARTIR DE SU OBRA CON FINES NO COMERCIALES, SIEMPRE Y CUANDO LE RECONOZCAN LA AUTORÍA Y SUS NUEVAS CREACIONES ESTÉN BAJO UNA LICENCIA CON LOS MISMOS TÉRMINOS.}

